\title{
Ultrahigh Green and Red Optical Gain Cross Sections from Solutions of Colloidal Quantum Well Heterostructures
}

\author{
Savas Delikanli, ${ }^{\S}$ Onur Erdem, ${ }^{\S}$ Furkan Isik, Hamed Dehghanpour Baruj, Farzan Shabani, \\ Huseyin Bilge Yagci, Emek Goksu Durmusoglu, and Hilmi Volkan Demir*
}

Cite This: J. Phys. Chem. Lett. 2021, 12, 2177-2182

Read Online

\section{ACCESS | Lill Metrics \& More | 回 Article Recommendations | (1) Supporting Information}

ABSTRACT: We demonstrate amplified spontaneous emission (ASE) in solution with ultralow thresholds of $30 \mu \mathrm{J} / \mathrm{cm}^{2}$ in red and of $44 \mu \mathrm{J} / \mathrm{cm}^{2}$ in green from engineered colloidal quantum well (CQW) heterostructures. For this purpose, CdSe/CdS core/crown CQWs, designed to hit the green region, and $\mathrm{CdSe} / \mathrm{CdS} @ \mathrm{Cd}_{x} \mathrm{Zn}_{1-x} \mathrm{~S}$ core/crown@gradient-alloyed shell CQWs, further tuned to reach the red region by shell alloying, were employed to achieve highperformance ASE in the visible range. The net modal gain of these CQWs reaches $530 \mathrm{~cm}^{-1}$ for the green and $201 \mathrm{~cm}^{-1}$ for the red, 2-3 orders of magnitude larger than those of colloidal quantum dots (QDs) in solution. To explain the root cause for ultrahigh gain coefficient in solution, we show for the first time that the gain cross sections of these CQWs is $\geq 3.3 \times 10^{-14}$ $\mathrm{cm}^{2}$ in the green and $\geq 1.3 \times 10^{-14} \mathrm{~cm}^{2}$ in the red, which are two orders of magnitude larger compared to those of CQDs.

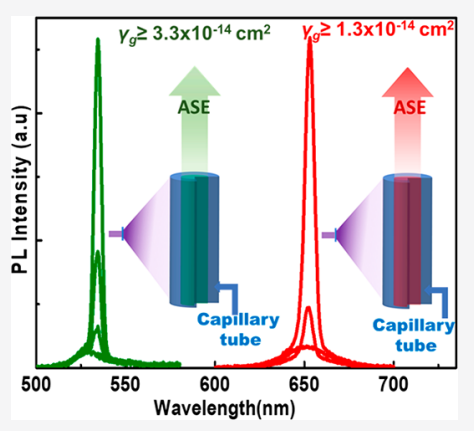

$\mathrm{C}$ olloidal quantum wells (CQWs) present an excellent platform as gain medium owing to their solution processability, wide tunability of emission color, ${ }^{1-4}$ giant oscillator strength, ${ }^{1,5}$ large gain cross section, ${ }^{6}$ slow Auger rates $^{7-9}$ and ultranarrow emission profiles. ${ }^{1,3,10}$ Especially the giant gain cross section and slow Auger recombination of CQWs make them extremely attractive candidates for optical gain studies and applications as these are the critical factors for satisfying the gain condition. ${ }^{11}$ Lasing and amplified spontaneous emission (ASE) with ultralow thresholds ${ }^{2,7,8,12-15}$ and large net modal gains ${ }^{7,14,16}$ from solid films of close-packed CQWs have been widely studied. Although a solution-based gain medium can be highly advantageous because of the enhanced photostability owing to constant replenishment of the gain medium by the flux of nanocrystals and flexibility of incorporation into optical cavities, there are only very few reports of optical gain in solution using colloidal nanocrystals. ${ }^{6,17-20}$ This is primarily due to the low concentration of gain media possible in solution, limiting the feasible levels of gain coefficients. While thin solid films of nanocrystals may lead to lower thresholds and larger modal gains due to the increased density of gain media, such films are more likely to suffer from surface roughness affecting the waveguiding and optical confinement, ${ }^{21-23}$ nonradiative losses due to Förster resonance energy transfer (FRET) and homo-FRET, ${ }^{24,25}$ and reproducibility of the identical samples.

In-solution optical gain can be achieved straightforwardly by employing a simple glass tube ${ }^{17}$ or a cuvette ${ }^{6,18}$ serving possibly as a cavity and a host along with highly concentrated nanocrystal solution serving as a gain medium. In this approach, uniform gain media in a cavity can be achieved robustly and reproducibly, and loss mechanisms, such as nonradiative homo-FRET and scattering of light due to aggregations of nanocrystals, are conveniently suppressed. Such a gain medium can be utilized in microfluidic networks to achieve on-chip lasing for optical sensing and detection. ${ }^{26-30}$ Despite the foreseeable advantages of CQWs, including the modal gain reaching levels 1 order of magnitude larger than those of the quantum dots and nanorods, ${ }^{16}$ slow Auger rates, ${ }^{7-9,31}$ and gain cross sections 3 orders of magnitude larger than the quantum dots, ${ }^{6}$ there is only one previously reported work on CQW-based optical gain in solution to date, which was achieved by multiphoton pumping using a cuvette-based Fabry-Perot resonator. ${ }^{6}$

Here, we present the achievement of in-solution ASE from green-emitting $\mathrm{CdSe} / \mathrm{CdS}$ core/crown CQWs and red CdSe/ $\mathrm{CdS} @ \mathrm{Cd}_{x} \mathrm{Zn}_{1-x} \mathrm{~S}$ core/crown@gradient-alloyed shell CQWs enabling ultralow thresholds of 44 and $30 \mu \mathrm{J} / \mathrm{cm}^{2}$, respectively, in solution. The net modal gains of green $\mathrm{CdSe} / \mathrm{CdS}$ core/ crown CQWs and red CdSe/CdS@ $\mathrm{Cd}_{x} \mathrm{Zn}_{1-x} \mathrm{~S}$ core/crown@ gradient-alloyed shell CQWs at a concentration of $1.7 \times 1016$ $\mathrm{cm}^{-3}$ reach $\sim 530$ and $\sim 201 \mathrm{~cm}^{-1}$, respectively. In addition, we report for the first time the gain cross section of these heterostructures of CQWs, which are found to be $\geq 3.3 \times 10^{-14}$ $\mathrm{cm}^{2}$ for the green-emitting $\mathrm{CdSe} / \mathrm{CdS}$ core/crown CQWs and $\geq 1.3 \times 10^{-14} \mathrm{~cm}^{2}$ for the red-emitting CdSe/CdS@Cd $\mathrm{Zn}_{1-x} \mathrm{~S}$

Received: December 29, 2020

Accepted: February 22, 2021

Published: February 25, 2021 
(a)
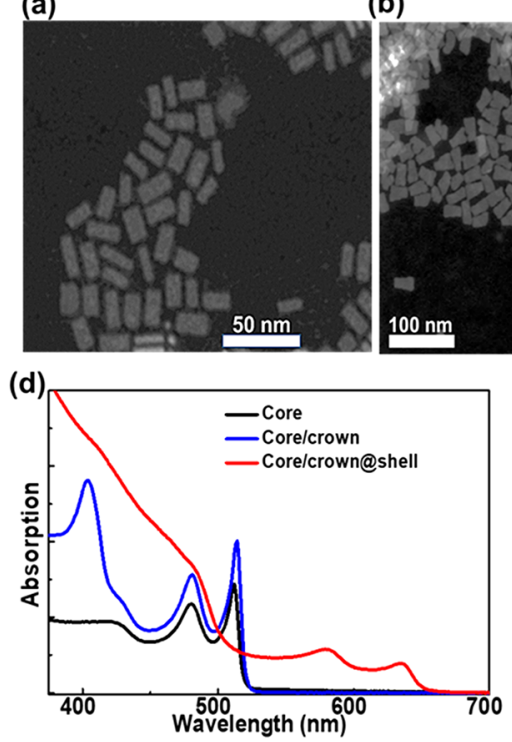

(b)

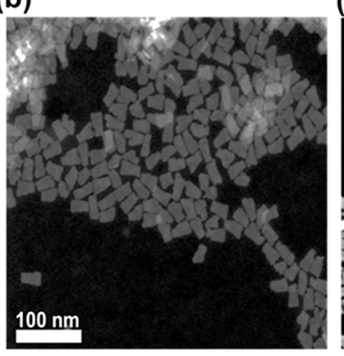

(e) (c)
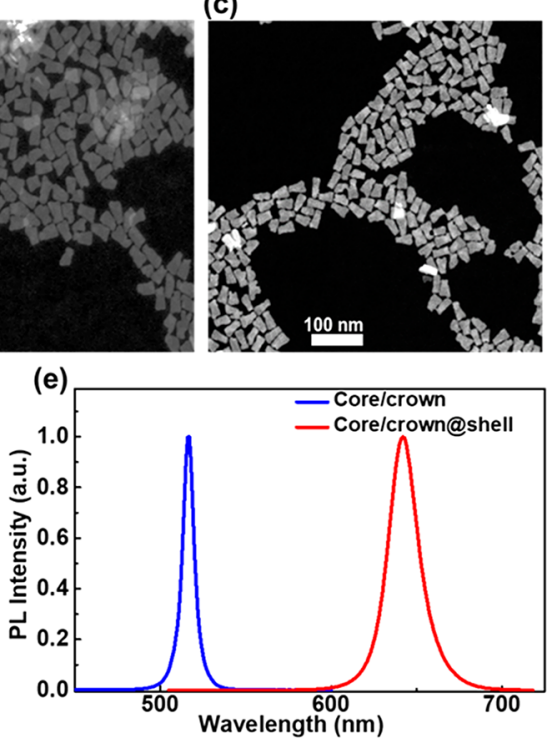

Figure 1. TEM images of (a) $4 \mathrm{ML} \mathrm{CdSe}$, (b) $4 \mathrm{ML} \mathrm{CdSe} / \mathrm{CdS}$ core/crown, and (c) $12 \mathrm{ML} \mathrm{CdSe} / \mathrm{CdS} @ \mathrm{Cd}_{x} \mathrm{Zn}_{1-x} \mathrm{~S}$ core/crown@gradientalloyed shell CQWs. (d) Absorption spectra of CdSe, CdSe/CdS core/crown, and CdSe/CdS@Cd $\mathrm{Zn}_{1-x} \mathrm{~S}$ core/crown@gradient-alloyed shell CQWs. (c) PL spectra of CdSe/CdS core/crown and CdSe/CdS@Cd $Z_{1-x} \mathrm{~S}$ core/crown@gradient-alloyed shell CQWs.
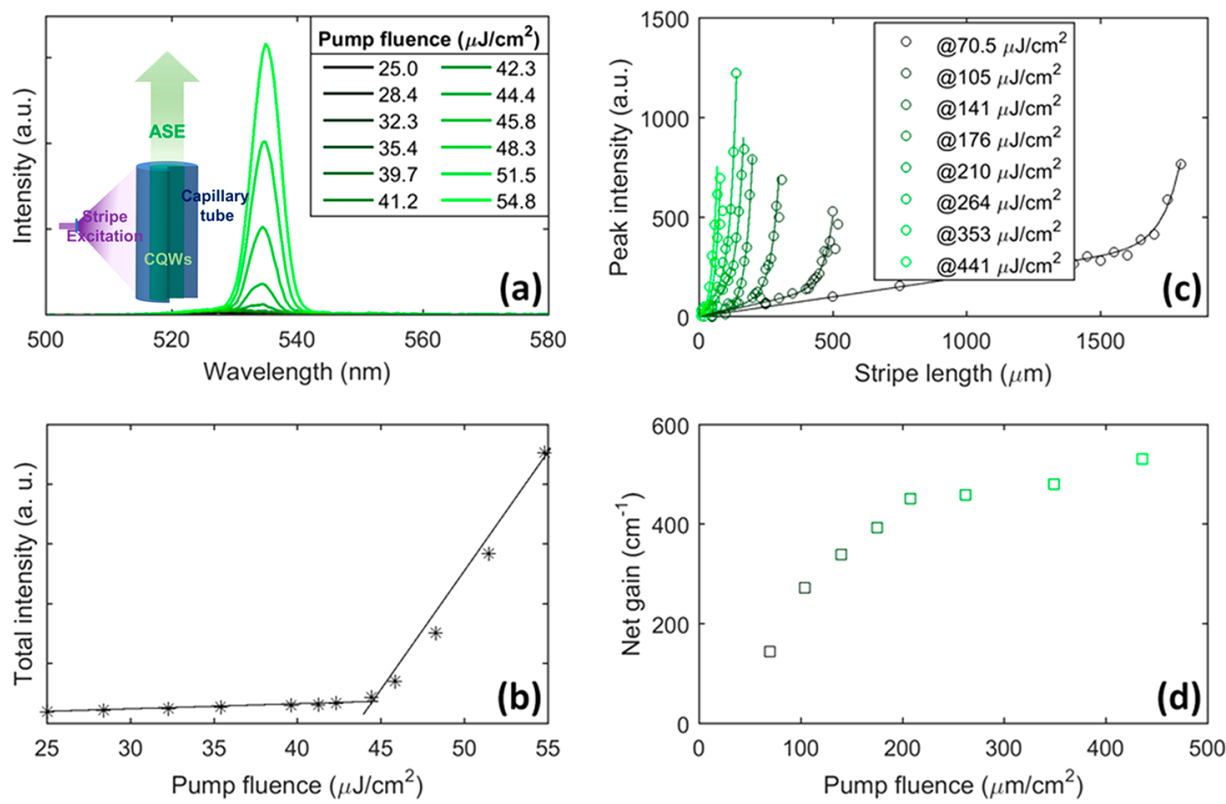

Figure 2. Optical gain performance of in-solution CdSe/CdS core/crown CQWs. (a) Collected optical spectra at different pump fluences. Inset of panel a shows a schematic of CQW solution in the capillary tube optically pumped using stripe geometry for ASE measurements. (b) Emission intensity as a function of the pump fluence. (c) Emission intensity as a function of the excitation stripe length. (d) Extracted net gain coefficients as a function of the pump fluence.

core/crown@gradient-alloyed shell CQWs. These obtained gain cross sections are 2 orders of magnitude larger than those of QDs ${ }^{11,17}$ and hence show the vast potential of CQWs as an in-solution optical gain medium.

For the purpose of systematic study of optical gain in solution, we synthesized $\mathrm{CdSe} / \mathrm{CdS}$ core/crown $\mathrm{CQWs}^{32}$ to hit the green spectral range and CdSe/CdS@ $\mathrm{Cd}_{x} \mathrm{Zn}_{1-x} \mathrm{~S}$ core/ crown@gradient-alloyed shell CQWs ${ }^{33}$ to reach the red range, both using high-quality 4-monolayer (ML) CdSe CQWs as the seeds. Transmission electron microscopy (TEM) images of the CdSe core CQWs and $\mathrm{CdSe} / \mathrm{CdS}$ core/crown CQWs are shown in panels $\mathrm{a}$ and $\mathrm{b}$ of Figure 1, respectively. The lateral area was measured to be $\sim 150 \mathrm{~nm}^{2}$ for the core CQWs and $\sim 350 \mathrm{~nm}^{2}$ for the core/crown CQWs.

To further obtain the CdSe/CdS@Cd $\mathrm{Zn}_{1-x} \mathrm{~S}$ core/crown@ gradient-alloyed shell CQWs, $4 \mathrm{ML}$ thick gradient alloyed shell of $\mathrm{Cd}_{x} \mathrm{Zn}_{1-x} \mathrm{~S}$ was grown on the previously synthesized CdSe/ CdS core/crown CQWs to push the emission peak toward red. ${ }^{33}$ Details of the synthesis of CQWs are provided in the Supporting Information. Owing to the gradient alloyed shell of $\mathrm{Cd}_{x} \mathrm{Zn}_{1-x} \mathrm{~S}$, charge carriers feel soft potential confinement in the vertical dimension, ${ }^{33}$ which is also highly desirable for optical gain applications because of the suppression of Auger 
recombination. ${ }^{34} \mathrm{~A}$ representative TEM image of these core/ crown@gradient-alloyed shell CQWs is presented in Figure 1c.

The absorption profiles of the CdSe core, CdSe/CdS core/ crown, and CdSe/CdS@Cd $\mathrm{Zn}_{1-x} \mathrm{~S}$ core/crown@gradientalloyed shell CQWs are given in Figure 1d. The absorption cross sections of $\mathrm{CdSe} / \mathrm{CdS}$ core/crown and CdSe/CdS@ $\mathrm{Cd}_{x} \mathrm{Zn}_{1-x} \mathrm{~S}$ core/crown@gradient-alloyed shell CQWs are 1.1 $\times 10^{-13} \mathrm{~cm}^{2}$ and $2.9 \times 10^{-13} \mathrm{~cm}^{2}$, respectively, at $400 \mathrm{~nm}$, which were calculated using a method provided in our previous publication. ${ }^{35}$ The intrinsic absorption coefficients $\mu_{i}(\lambda)$ of the $\mathrm{CdSe} / \mathrm{CdS}$ core/crown and $\mathrm{CdSe} / \mathrm{CdS} @ \mathrm{Cd}_{x} \mathrm{Zn}_{1-x} \mathrm{~S}$ core/ crown@gradient-alloyed shell CQWs, which are obtained by dividing the absorption cross sections at the wavelength of interest by the physical volume of the CQWs, are $1.96 \times 10^{5}$ and $2.1 \times 10^{4} \mathrm{~cm}^{-1}$, respectively, at their heavy hole absorption peak. It is worth mentioning that the intrinsic absorption is an important parameter showing the gain capability of a material. $^{36}$ The photoluminescence (PL) spectra of CdSe/ $\mathrm{CdS}$ core/crown and CdSe/CdS@Cd $\mathrm{Zn}_{1-x} \mathrm{~S}$ core/crown@ gradient-alloyed shell CQWs in solution are shown in Figure 1e. The PL peak of the core/crown CQWs is at $\sim 517 \mathrm{~nm}$ (in solution) with a full width at half-maximum (fwhm) of $8 \mathrm{~nm}$, while that of CdSe/CdS@ $\mathrm{Cd}_{x} \mathrm{Zn}_{1-x} \mathrm{~S}$ core/crown@gradientalloyed shell CQWs (in solution) is at $\sim 642 \mathrm{~nm}$ with a fwhm of $\sim 21 \mathrm{~nm}$.

To explore the ASE performance of our CQWs in solution, CQWs dispersed in toluene were inserted into a capillary tube having an inner diameter of $300 \mu \mathrm{m}$ with the help of capillary force. The higher boiling point (compared to most nonpolar solvents, such as commonly used hexane), the lower volatility, and the relatively high refractive index of toluene make it our choice of solvent. Figure 2a shows pump-fluence dependence of the ASE spectra from CdSe/CdS core/crown CQWs. The sharp ASE peak can be observed at $534.2 \mathrm{~nm}$ with a fwhm of $4.3 \mathrm{~nm}$ at room temperature on the red side of the spontaneous emission for the pump fluences above the threshold. This red shift of ASE $(\sim 5 \mathrm{~nm})$ with respect to the spontaneous emission confirms multiexcitonic gain. ${ }^{37}$ This sort of red shift in ASE is desirable for optical gain because this hinders self-absorption. ${ }^{7,38}$ Total emission intensity as a function of the pump fluence for the green-emitting CQWs is presented in Figure 2b. Using this data, the ASE threshold is calculated to be $44 \mu \mathrm{J} / \mathrm{cm}^{2}$, which is lower than any previously reported ASE threshold in solution for nanocrystals. Previously, the best reported threshold in solution for colloidal nanocrystals was $\sim 105 \mu \mathrm{J} / \mathrm{cm}^{2}$ from $\mathrm{CsPbBr}_{3}$ perovskite nanocrystals. $^{18}$

We used the variable-stripe-length (VSL) $\operatorname{method}^{39}$ to determine the modal gain of CQWs in solution at various pump fluences higher than the optical gain threshold. Figure $2 c$ displays total emission intensities at different pump fluences as a function of the stripe length. Figure $2 \mathrm{~d}$ presents the pumpdependent net modal gain coefficient at various pump fluences obtained from the fittings presented in Figure 2c. As can be seen in Figure 2d, the net modal gain increases linearly at lower fluences above the threshold and saturates around $\sim 530 \mathrm{~cm}^{-1}$. Although these net modal gains are nearly 1 order of magnitude lower than those of their solid films ${ }^{7,16}$ because of lower concentration of CQWs, these net modal gains are similar to the net modal gains of solid films of quantum dots and nanorods ${ }^{40-43}$ and $2-3$ orders of magnitude larger than that of QDs in solution. ${ }^{17}$

The gain cross section per CQW $\left(\gamma_{\mathrm{g}}\right)$ can be calculated by

$$
g_{\text {net }}=C \times \Gamma \times \gamma_{\mathrm{g}}-\alpha
$$

where $C$ is the concentration of CQWs, $\Gamma$ the optical confinement factor, and $\alpha$ the optical loss coefficient. ${ }^{6}$ In our case, $C$ is $\sim 1.7 \times 10^{16} \mathrm{~cm}^{-3}(\sim 28.3 \mu \mathrm{M})$ in toluene; $g_{\text {net }}$ is known at different pump fluences as presented in Figure $2 \mathrm{~d}$, and $\Gamma$ is calculated by numerically solving the supported optical modes, as will be discussed next. Here, we assume that the net modal gain is close to the modal gain $(g)$ for optical gain in solution. ${ }^{6}$ In fact, our net modal gain levels $\left(530 \mathrm{~cm}^{-1}\right.$ for CdSe/CdS core/crown CQWs and $201 \mathrm{~cm}^{-1}$ for CdSe/ $\mathrm{CdS} @ \mathrm{Cd}_{x} \mathrm{Zn}_{1-x} \mathrm{~S}$ core/crown@ shell CQWs) are much larger than even the loss coefficient from films of CQWs $(\sim 10$ $\left.\mathrm{cm}^{-1}\right),{ }^{22}$ which are expected to suffer larger loss in film compared to in solution. In addition, we added the term $\Gamma$ into the eq 1 to account for the optical confinement factor in the active optical gain media.

Here, $\Gamma$ was calculated by obtaining the electrical field distribution in our system. $\Gamma$ for a mode is defined as the ratio of the modal power inside the excited portion of the gain media to the total modal power:

$$
\begin{aligned}
\Gamma= & \int_{\text {excited gain region }} \frac{1}{2} \operatorname{Re}\left\{\vec{E} \times \vec{H}^{*}\right\} \cdot \hat{z} \mathrm{~d} x \mathrm{~d} y / \\
& \int_{-\infty}^{\infty} \frac{1}{2} \operatorname{Re}\left\{\vec{E} \times \vec{H}^{*}\right\} \cdot \hat{z} \mathrm{~d} x \mathrm{~d} y
\end{aligned}
$$

where $\vec{E}$ and $\vec{H}$ are the electric and magnetic fields of the specified mode, respectively. The gain cross section $\left(\gamma_{\mathrm{g}}\right)$ can be found by computing $\Gamma$ for the modes that are guided and confined. The modal power distributions for the first four modes $\left(\mathrm{HE}_{11}, \mathrm{HE}_{21}, \mathrm{HE}_{31}\right.$, and $\left.\mathrm{HE}_{12}\right)$ for $\mathrm{CdSe} / \mathrm{CdS}$ core/ crown CQWs are presented in Figure $3 \mathrm{a}-\mathrm{d}$. The confinement factor peaks at 0.94 for $\mathrm{HE}_{11}$ because of strong overlap with the excitation. On the basis of this maximal $\Gamma$, we computed the minimum gain cross section of $\mathrm{CdSe} / \mathrm{CdS}$ core/crown CQWs using the modal gain value of $530 \mathrm{~cm}^{-1}$ and the concentration of $1.7 \times 10^{16} \mathrm{~cm}^{-3}$. The obtained value of gain cross section for $\mathrm{CdSe} / \mathrm{CdS}$ core/crown CQWs is $\geq 3.3 \times 10^{-14} \mathrm{~cm}^{2}$, which is 2 orders of magnitude larger than the gain cross section of $\mathrm{QDs}^{17}$ having gain profiles saturating approximately twice of their thresholds, suggesting a limited number of carrier species contributing gain in the case of QDs. We attribute this extremely large gain cross section of CQWs to the high number of density of states in the CQWs.

Figure $4 \mathrm{a}$ presents the pump-fluence dependence of ASE spectra from the solution of $\mathrm{CdSe} / \mathrm{CdS} @ \mathrm{Cd}_{x} \mathrm{Zn}_{1-x} \mathrm{~S}$ core/ crown@gradient-alloyed shell CQWs. In these CQWs, the spontaneous emission peaks at $651.9 \mathrm{~nm}$ with a fwhm of 29.0 $\mathrm{nm}$. As the pump intensity increases, a sharp peak emerges at $653.0 \mathrm{~nm}$ with a fwhm of $6.0 \mathrm{~nm}$ and ultimately surpasses the PL spectrum at stronger pump fluences. The reduced red shift of ASE in this sample compared to that of the core/crown one can be attributed to the multiexcitonic gain with quasi-type-II band alignment profile. Total emission intensity as a function of the pump fluence is given in Figure $4 \mathrm{~b}$. The ASE threshold for these red-emitting CQWs is $30 \mu \mathrm{J} / \mathrm{cm}^{2}$, which is lower than that of the green core/crown CQWs. This reduction in the gain threshold can be attributed to the further efficient suppression of Auger recombination in these alloyed-shell CQW heterostructures compared to the core/crown CQWs as a result of the graded alloying providing a soft confinement potential. $^{7,34}$ Figure $4 \mathrm{c}$ shows total emission intensities at 

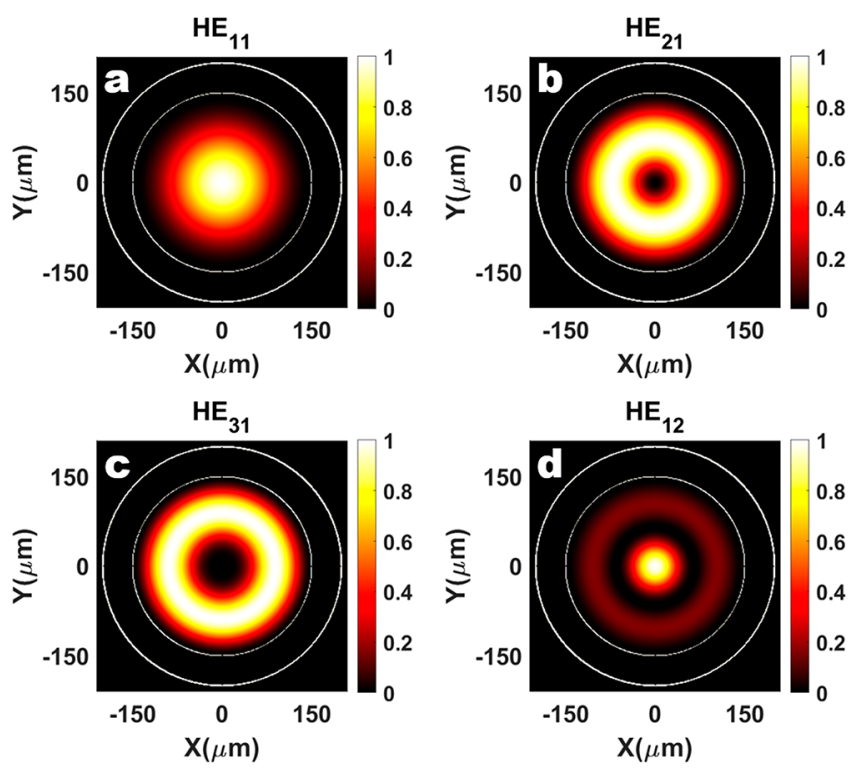

Figure 3. $(\mathrm{a}-\mathrm{d})$ Modal guided power distributions at $534 \mathrm{~nm}$ for the first four modes. The boundaries of the fiber are indicated with white concentric rings with the large ring $(r=200 \mu \mathrm{m})$ corresponding to the air-cladding boundary and the small ring $(r=150 \mu \mathrm{m})$ corresponding to the cladding-core boundary. Because of the large fiber parameter $V$ and a similar refractive index of our CQW solutions, the calculated profiles at 653 and $534 \mathrm{~nm}$ are practically identical. The reason behind the near-unity confinement factor for $\mathrm{HE}_{11}$ is easily understood from the regional overlap between mode and the excitation (see Figure S3).

different pump fluences as a function of the stripe length, and the net modal gain coefficients at various pump fluences obtained from the fittings given in Figure $4 c$ are presented in Figure $4 \mathrm{~d}$. As shown in Figure $4 \mathrm{~d}$, the net modal gain saturates around $201 \mathrm{~cm}^{-1}$ at a pump fluence of $\sim 350 \mu \mathrm{J} / \mathrm{cm}^{2}$. This maximum net modal gain from these red CQWs in solution is 2 orders of magnitude higher than that of $\mathrm{CdZnS} / \mathrm{ZnS}$ alloyedcore/shell QDs ${ }^{17}$ and similar to that of core/shell CQWs. ${ }^{6}$ Modal characteristics of the red-emitting CQWs are identical to $\mathrm{CdSe} / \mathrm{CdS}$ core/crown CQWs because of the large fiber parameter $V$ and similar refractive index of CQW solutions, and hence, both CQW solutions share the same $\Gamma$ value of 0.94 at the ASE peak wavelength. For these red CQWs, the gain cross section is found to be $\geq 1.3 \times 10^{-14} \mathrm{~cm}^{2}$. However, the gain cross section of these core/crown@gradient-alloyed shell CQWs is lower than that of core/crown CQWs $\left(\sim 3.3 \times 10^{-14}\right.$ $\left.\mathrm{cm}^{2}\right)$. This is also reflected by the lower absorption coefficient of the core/crown@gradient-alloyed shell CQWs compared to the core/crown CQWs. This can be explained by the quasi type-II band alignment of thicker core/crown@gradientalloyed shell CQWs, which effectively reduces the oscillator strength of the transitions in the core/crown@gradient-alloyed shell CQWs. On the other hand, the intriguingly lower threshold of the core/crown@gradient-alloyed shell CQWs is dominantly dictated by their larger absorption cross section at the excitation wavelength and stronger suppression of Auger recombination as compared to the core/crown CQWs.

In summary, we demonstrated ASE in solution using CdSe/ $\mathrm{CdS}$ core/crown CQWs designed to emit in green and CdSe/ $\mathrm{CdS} @ \mathrm{Cd}_{x} \mathrm{Zn}_{1-x} \mathrm{~S}$ core/crown@gradient-alloyed shell CQWs tuned to emit in red dispersed in toluene with ultralow thresholds of 44 and $30 \mu \mathrm{J} / \mathrm{cm}^{2}$, respectively. The net modal gain coefficients of these green and red CQWs reach $\sim 530$ and $\sim 201 \mathrm{~cm}^{-1}$, respectively, at a concentration of $\sim 1.7 \times 10^{16}$ $\mathrm{cm}^{-3}$, which are 2-3 orders of magnitude larger than that of QDs in solution. ${ }^{17}$ The gain cross sections of the green CdSe/ $\mathrm{CdS}$ core/crown CQWs and the red CdSe/CdS@Cd $\mathrm{Zn}_{1-x} \mathrm{~S}$ core/crown@gradient-alloyed shell CQWs are $\geq 3.3 \times 10^{-14}$ $\mathrm{cm}^{2}$ and $\geq 1.3 \times 10^{-14} \mathrm{~cm}^{2}$, respectively. The obtained gain cross sections are 2 orders of magnitude larger than the gain cross section of QDs and can be attributed to the higher
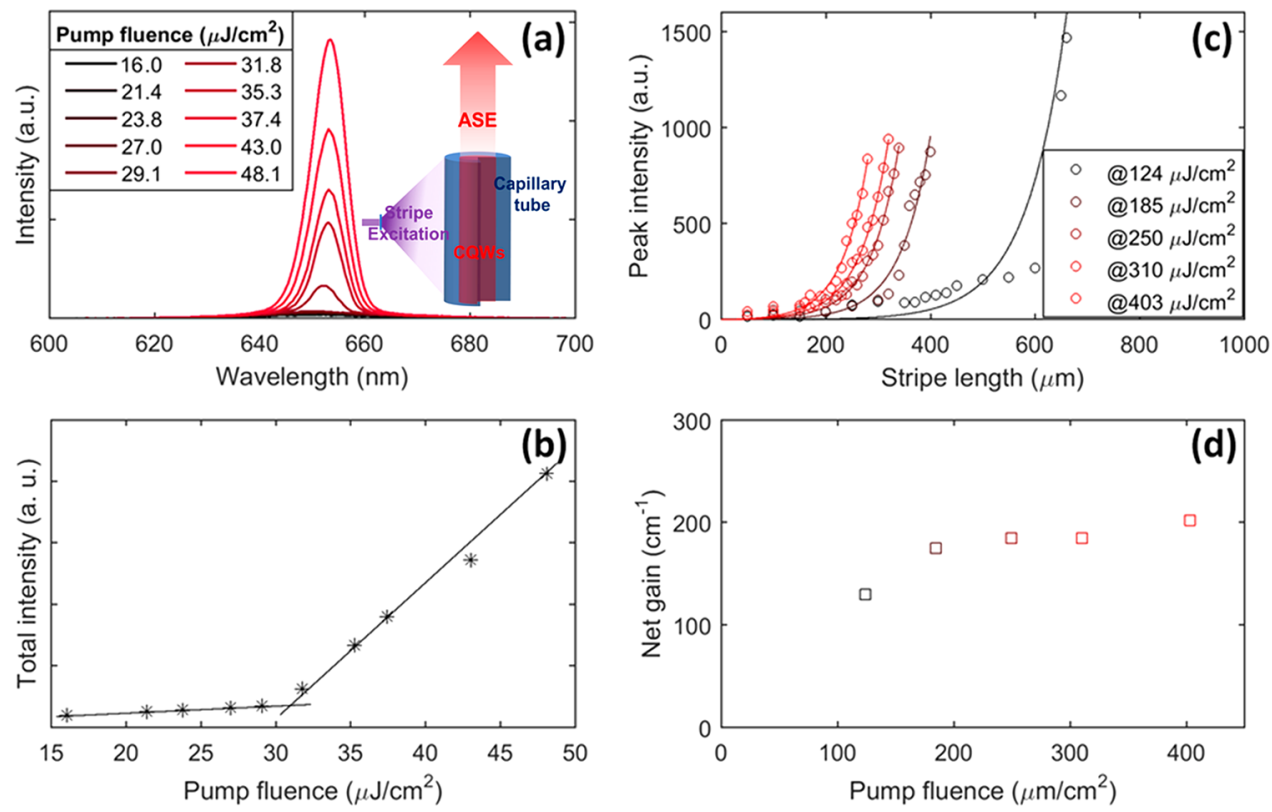

Figure 4. Optical gain performance of in-solution CdSe/CdS@Cd $\mathrm{Zn}_{1-x} \mathrm{~S}$ core/crown@gradient-alloyed shell CQWs. (a) Collected optical spectra at different pump fluences. The inset of panel a displays a schematic of CQW solution in the capillary tube pumped using stripe geometry for ASE measurements. (b) Emission intensity as a function of the pump fluence. (c) Emission intensity as a function of the excitation stripe length. (d) Extracted net gain coefficients as a function of the pump fluence. 
available number of density of states in CQWs. These solutionprocessed CQWs with their exceptional properties as a solution-based optical gain medium present extraordinary opportunities for the design and implementation of solutionbased lasers, which can be integrated conveniently and intimately into microfluidic devices designed for sensing and imaging applications.

\section{ASSOCIATED CONTENT}

\section{SI Supporting Information}

The Supporting Information is available free of charge at https://pubs.acs.org/doi/10.1021/acs.jpclett.0c03836.

Synthesis of CdSe/CdS core/crown CQWs and CdSe/ $\mathrm{CdS} @ \mathrm{Cd}_{x} \mathrm{Zn}_{1-x} \mathrm{~S}$ core/crown@gradient-alloyed shell CQWs, time-resolved PL measurements, details of the optical gain experiments, and numerical calculations for optical confinement factor (PDF)

\section{AUTHOR INFORMATION}

\section{Corresponding Author}

Hilmi Volkan Demir - Department of Electrical and Electronics Engineering, Department of Physics, UNAM Institute of Materials Science and Nanotechnology, Bilkent University, Ankara 06800, Turkey; Luminous! Center of Excellence for Semiconductor Lighting and Displays, School of Electrical and Electronic Engineering, School of Physical and Materials Sciences, Nanyang Technological University, Singapore 639798, Singapore; 이이.org/0000-00031793-112X; Email: volkan@bilkent.edu.tr, hvdemir@ ntu.edu.sg

\section{Authors}

Savas Delikanli - Department of Electrical and Electronics Engineering, Department of Physics, UNAM - Institute of Materials Science and Nanotechnology, Bilkent University, Ankara 06800, Turkey; Luminous! Center of Excellence for Semiconductor Lighting and Displays, School of Electrical and Electronic Engineering, School of Physical and Materials Sciences, Nanyang Technological University, Singapore 639798, Singapore; (i) orcid.org/0000-0002-0613-8014

Onur Erdem - Department of Electrical and Electronics Engineering, Department of Physics, UNAM - Institute of Materials Science and Nanotechnology, Bilkent University, Ankara 06800, Turkey

Furkan Isik - Department of Electrical and Electronics Engineering, Department of Physics, UNAM - Institute of Materials Science and Nanotechnology, Bilkent University, Ankara 06800, Turkey

Hamed Dehghanpour Baruj - Department of Electrical and Electronics Engineering, Department of Physics, UNAM Institute of Materials Science and Nanotechnology, Bilkent University, Ankara 06800, Turkey

Farzan Shabani - Department of Electrical and Electronics Engineering, Department of Physics, UNAM - Institute of Materials Science and Nanotechnology, Bilkent University, Ankara 06800, Turkey

Huseyin Bilge Yagci - Department of Electrical and Electronics Engineering, Department of Physics, UNAM Institute of Materials Science and Nanotechnology, Bilkent University, Ankara 06800, Turkey

Emek Goksu Durmusoglu - Luminous! Center of Excellence for Semiconductor Lighting and Displays, School of Electrical and Electronic Engineering, School of Physical and Materials Sciences, Nanyang Technological University, Singapore

639798, Singapore; 이이.orcid/0000-0001-6840-8342

Complete contact information is available at:

https://pubs.acs.org/10.1021/acs.jpclett.0c03836

\section{Author Contributions}

${ }^{\S}$ S.D. and O.E contributed equally.

\section{Notes}

The authors declare no competing financial interest.

\section{ACKNOWLEDGMENTS}

The authors gratefully acknowledge the financial support in part from Singapore National Research Foundation under the programs of NRF-NRFI2016-08, NRF-CRP14-2014-03 and the Science and the Singapore Agency for Science, Technology and Research (A*STAR) SERC Pharos Program under Grant No. 152-73-00025 and in part from TUBITAK 115F297, 117E713, and 119N343. H.V.D. also gratefully acknowledges support from TUBA. O.E. acknowledges support from TUBITAK BIDEB.

\section{REFERENCES}

(1) Ithurria, S.; Tessier, M. D.; Mahler, B.; Lobo, R. P. S. M.; Dubertret, B.; Efros, A. L. Colloidal Nanoplatelets with TwoDimensional Electronic Structure. Nat. Mater. 2011, 10, 936.

(2) Sharma, M.; Delikanli, S.; Demir, H. V. Two-Dimensional CdSeBased Nanoplatelets: Their Heterostructures, Doping, Photophysical Properties, and Applications. Proc. IEEE 2020, 108, 655-675.

(3) Ithurria, S.; Dubertret, B. Quasi 2d Colloidal CdSe Platelets with Thicknesses Controlled at the Atomic Level. J. Am. Chem. Soc. 2008, 130, 16504-16505.

(4) Delikanli, S.; Guzelturk, B.; Hernández-Martínez, P. L.; Erdem, T.; Kelestemur, Y.; Olutas, M.; Akgul, M. Z.; Demir, H. V. Continuously Tunable Emission in Inverted Type-I CdS/CdSe Core/Crown Semiconductor Nanoplatelets. Adv. Funct. Mater. 2015, 25, 4282-4289.

(5) Naeem, A.; Masia, F.; Christodoulou, S.; Moreels, I.; Borri, P.; Langbein, W. Giant Exciton Oscillator Strength and Radiatively Limited Dephasing in Two-Dimensional Platelets. Phys. Rev. B: Condens. Matter Mater. Phys. 2015, 91, 121302.

(6) Li, M.; Zhi, M.; Zhu, H.; Wu, W.-Y.; Xu, Q.-H.; Jhon, M. H.; Chan, Y. Ultralow-Threshold Multiphoton-Pumped Lasing from Colloidal Nanoplatelets in Solution. Nat. Commun. 2015, 6, 8513.

(7) Taghipour, N.; Delikanli, S.; Shendre, S.; Sak, M.; Li, M.; Isik, F.; Tanriover, I.; Guzelturk, B.; Sum, T. C.; Demir, H. V. Sub-Single Exciton Optical Gain Threshold in Colloidal Semiconductor Quantum Wells with Gradient Alloy Shelling. Nat. Commun. 2020, $11,3305$.

(8) Altintas, Y.; Gungor, K.; Gao, Y.; Sak, M.; Quliyeva, U.; Bappi, G.; Mutlugun, E.; Sargent, E. H.; Demir, H. V. Giant Alloyed Hot Injection Shells Enable Ultralow Optical Gain Threshold in Colloidal Quantum Wells. ACS Nano 2019, 13, 10662-10670.

(9) Kunneman, L. T.; Tessier, M. D.; Heuclin, H.; Dubertret, B.; Aulin, Y. V.; Grozema, F. C.; Schins, J. M.; Siebbeles, L. D. A. Bimolecular Auger Recombination of Electron-Hole Pairs in TwoDimensional Cdse and $\mathrm{CdSe} / \mathrm{CdZnS}$ Core/Shell Nanoplatelets. J. Phys. Chem. Lett. 2013, 4, 3574-3578.

(10) Hazarika, A.; Fedin, I.; Hong, L.; Guo, J.; Srivastava, V.; Cho, W.; Coropceanu, I.; Portner, J.; Diroll, B. T.; Philbin, J. P.; et al. Colloidal Atomic Layer Deposition with Stationary Reactant Phases Enables Precise Synthesis of "Digital" II-VI Nano-Heterostructures with Exquisite Control of Confinement and Strain. J. Am. Chem. Soc. 2019, 141, 13487-13496.

(11) Klimov, V. I.; Mikhailovsky, A. A.; Xu, S.; Malko, A.; Hollingsworth, J. A.; Leatherdale, C. A.; Eisler, H.-J.; Bawendi, M. 
G. Optical Gain and Stimulated Emission in Nanocrystal Quantum Dots. Science 2000, 290, 314-317.

(12) Dede, D.; Taghipour, N.; Quliyeva, U.; Sak, M.; Kelestemur, Y.; Gungor, K.; Demir, H. V. Highly Stable Multicrown Heterostructures of Type-II Nanoplatelets for Ultralow Threshold Optical Gain. Chem. Mater. 2019, 31, 1818-1826.

(13) She, C.; Fedin, I.; Dolzhnikov, D. S.; Dahlberg, P. D.; Engel, G. S.; Schaller, R. D.; Talapin, D. V. Red, Yellow, Green, and Blue Amplified Spontaneous Emission and Lasing Using Colloidal CdSe Nanoplatelets. ACS Nano 2015, 9, 9475-9485.

(14) She, C.; Fedin, I.; Dolzhnikov, D. S.; Demortière, A.; Schaller, R. D.; Pelton, M.; Talapin, D. V. Low-Threshold Stimulated Emission Using Colloidal Quantum Wells. Nano Lett. 2014, 14, 2772-2777.

(15) Kelestemur, Y.; Shynkarenko, Y.; Anni, M.; Yakunin, S.; De Giorgi, M. L.; Kovalenko, M. V. Colloidal CdSe Quantum Wells with Graded Shell Composition for Low-Threshold Amplified Spontaneous Emission and Highly Efficient Electroluminescence. ACS Nano 2019, 13, 13899-13909.

(16) Guzelturk, B.; Pelton, M.; Olutas, M.; Demir, H. V. Giant Modal Gain Coefficients in Colloidal Ii-Vi Nanoplatelets. Nano Lett. 2019, 19, 277-282.

(17) Wang, Y.; Leck, K. S.; Ta, V. D.; Chen, R.; Nalla, V.; Gao, Y.; He, T.; Demir, H. V.; Sun, H. Blue Liquid Lasers from Solution of CdZnS/ZnS Ternary Alloy Quantum Dots with Quasi-Continuous Pumping. Adv. Mater. 2015, 27, 169-175.

(18) Tan, M. J. H.; Wang, Y.; Chan, Y. Solution-Based Green Amplified Spontaneous Emission from Colloidal Perovskite Nanocrystals Exhibiting High Stability. Appl. Phys. Lett. 2019, 114, 183101.

(19) Schäfer, J.; Mondia, J. P.; Sharma, R.; Lu, Z. H.; Susha, A. S.; Rogach, A. L.; Wang, L. J. Quantum Dot Microdrop Laser. Nano Lett. 2008, 8, 1709-1712.

(20) Kazes, M.; Lewis, D. Y.; Ebenstein, Y.; Mokari, T.; Banin, U. Lasing from Semiconductor Quantum Rods in a Cylindrical Microcavity. Adv. Mater. 2002, 14, 317-321.

(21) Lee, K. K.; Lim, D. R.; Luan, H.-C.; Agarwal, A.; Foresi, J.; Kimerling, L. C. Effect of Size and Roughness on Light Transmission in a $\mathrm{Si} / \mathrm{SiO}_{2}$ Waveguide: Experiments and Model. Appl. Phys. Lett. 2000, 77, 1617-1619.

(22) Sak, M.; Taghipour, N.; Delikanli, S.; Shendre, S.; Tanriover, I.; Foroutan, S.; Gao, Y.; Yu, J.; Yanyan, Z.; Yoo, S.; et al. Coreless FiberBased Whispering-Gallery-Mode Assisted Lasing from Colloidal Quantum Well Solids. Adv. Funct. Mater. 2020, 30 (1), 1907417.

(23) Abécassis, B.; Tessier, M. D.; Davidson, P.; Dubertret, B. SelfAssembly of CdSe Nanoplatelets into Giant Micrometer-Scale Needles Emitting Polarized Light. Nano Lett. 2014, 14, 710-715.

(24) Guzelturk, B.; Erdem, O.; Olutas, M.; Kelestemur, Y.; Demir, H. V. Stacking in Colloidal Nanoplatelets: Tuning Excitonic Properties. ACS Nano 2014, 8, 12524-12533.

(25) Erdem, O.; Olutas, M.; Guzelturk, B.; Kelestemur, Y.; Demir, H. V. Temperature-Dependent Emission Kinetics of Colloidal Semiconductor Nanoplatelets Strongly Modified by Stacking. J. Phys. Chem. Lett. 2016, 7, 548-554.

(26) Monat, C.; Domachuk, P.; Eggleton, B. J. Integrated Optofluidics: A New River of Light. Nat. Photonics 2007, 1, 106-114. (27) Kim, D.; Popescu, P.; Harfouche, M.; Sendowski, J.; Dimotsantou, M.-E.; Flagan, R. C.; Yariv, A. On-Chip Integrated Differential Optical Microring Refractive Index Sensing Platform Based on a Laminar Flow Scheme. Opt. Lett. 2015, 40, 4106-4109.

(28) Sun, Y.; Fan, X. Distinguishing DNA by Analog-to-Digital-Like Conversion by Using Optofluidic Lasers. Angew. Chem., Int. Ed. 2012, 51, 1236-1239.

(29) Pang, L.; Chen, H. M.; Freeman, L. M.; Fainman, Y. Optofluidic Devices and Applications in Photonics, Sensing and Imaging. Lab Chip 2012, 12, 3543-3551.

(30) Fan, X.; White, I. M. Optofluidic Microsystems for Chemical and Biological Analysis. Nat. Photonics 2011, 5, 591-597.

(31) Diroll, B. T.; Talapin, D. V.; Schaller, R. D. Violet-to-Blue Gain and Lasing from Colloidal CdS Nanoplatelets: Low-Threshold
Stimulated Emission Despite Low Photoluminescence Quantum Yield. ACS Photonics 2017, 4, 576-583.

(32) Tessier, M. D.; Spinicelli, P.; Dupont, D.; Patriarche, G.; Ithurria, S.; Dubertret, B. Efficient Exciton Concentrators Built from Colloidal Core/Crown CdSe/CdS Semiconductor Nanoplatelets. Nano Lett. 2014, 14, 207-213.

(33) Shendre, S.; Delikanli, S.; Li, M.; Dede, D.; Pan, Z.; Ha, S. T.; Fu, Y. H.; Hernández-Martínez, P. L.; Yu, J.; Erdem, O.; et al. Ultrahigh-Efficiency Aqueous Flat Nanocrystals of CdSe/CdS@ $\mathrm{Cd}_{1-\mathrm{X}} \mathrm{Zn}_{\mathrm{x}} \mathrm{S}$ Colloidal Core/Crown@Alloyed-Shell Quantum Wells. Nanoscale 2019, 11, 301-310.

(34) Cragg, G. E.; Efros, A. L. Suppression of Auger Processes in Confined Structures. Nano Lett. 2010, 10, 313-317.

(35) Delikanli, S.; Yu, G.; Yeltik, A.; Bose, S.; Erdem, T.; Yu, J.; Erdem, O.; Sharma, M.; Sharma, V. K.; Quliyeva, U.; et al. Ultrathin Highly Luminescent Two-Monolayer Colloidal CdSe Nanoplatelets. Adv. Funct. Mater. 2019, 29, 1901028.

(36) Bisschop, S.; Geiregat, P.; Aubert, T.; Hens, Z. The Impact of Core/Shell Sizes on the Optical Gain Characteristics of CdSe/CdS Quantum Dots. ACS Nano 2018, 12, 9011-9021.

(37) Pietryga, J. M.; Park, Y.-S.; Lim, J.; Fidler, A. F.; Bae, W. K.; Brovelli, S.; Klimov, V. I. Spectroscopic and Device Aspects of Nanocrystal Quantum Dots. Chem. Rev. 2016, 116, 10513-10622.

(38) Dang, C.; Lee, J.; Breen, C.; Steckel, J. S.; Coe-Sullivan, S.; Nurmikko, A. Red, Green and Blue Lasing Enabled by Single-Exciton Gain in Colloidal Quantum Dot Films. Nat. Nanotechnol. 2012, 7, 335-339.

(39) Shaklee, K. L.; Nahory, R. E.; Leheny, R. F. Optical Gain in Semiconductors. J. Lumin. 1973, 7, 284-309.

(40) Lin, C. H.; Lafalce, E.; Jung, J.; Smith, M. J.; Malak, S. T.; Aryal, S.; Yoon, Y. J.; Zhai, Y.; Lin, Z.; Vardeny, Z. V.; et al. Core/AlloyedShell Quantum Dot Robust Solid Films with High Optical Gains. ACS Photonics 2016, 3, 647-658.

(41) Malko, A. V.; Mikhailovsky, A. A.; Petruska, M. A.; Hollingsworth, J. A.; Htoon, H.; Bawendi, M. G.; Klimov, V. I. From Amplified Spontaneous Emission to Microring Lasing Using Nanocrystal Quantum Dot Solids. Appl. Phys. Lett. 2002, 81, 13031305.

(42) Liao, Y.; Xing, G.; Mishra, N.; Sum, T. C.; Chan, Y. Low Threshold, Amplified Spontaneous Emission from Core-Seeded Semiconductor Nanotetrapods Incorporated into a Sol-Gel Matrix. Adv. Mater. 2012, 24, 159-164.

(43) Kazes, M.; Oron, D.; Shweky, I.; Banin, U. Temperature Dependence of Optical Gain in CdSe/ZnS Quantum Rods. J. Phys. Chem. C 2007, 111, 7898-7905. 